\title{
La enseñanza basada en la reflexión: la prefijación en Educación Secundaria
}

\section{Teaching based on reflexion: prefixation in Secondary Education}

\section{Irene del Rey Carchenilla}

Universidad Autónoma de Madrid, Grupo MORFONET irene.reyc@estudiante.uam.es

ORCID ID: https://orcid.org/0000-0001-7233-0533

DOI: $10.17398 / 1988-8430.35 .1 .241$

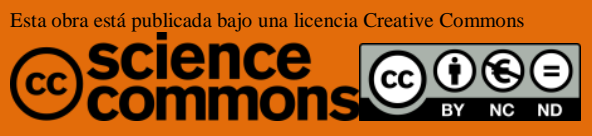

Fecha de recepción: 19/04/2021

Fecha de aceptación: 15/11/2021 ACCESS

Rey Carchenilla, I. del. (2022). La enseñanza basada en la reflexión: la prefijación en Educación Secundaria. Tejuelo, 35(1), 241-265.

Doi: https://doi.org/10.17398/1988-8430.35.1.241 
Resumen: En este artículo se analizan los problemas que presentan la clasificación de los prefijos y los ejercicios sobre prefijación en los libros de secundaria. La asignatura de Lengua castellana y Literatura debe aportar al alumno herramientas y conocimientos necesarios para que pueda desenvolverse en cualquier situación comunicativa. Uno de estos conocimientos es la gramática. Se comenta en estas páginas que los ejercicios tanto de identificación y etiquetado de prefijos como los de producción no favorecen la capacidad de los estudiantes para observar, argumentar, reflexionar y, en concreto, comprender las estructuras morfológicas. Nos centramos en el estudio de los prefijos en el ámbito de la Enseñanza Secundaria Obligatoria a través de una muestra de actividades que favorecen el desarrollo de la capacidad crítica del alumno para observar fenómenos morfológicos y formular generalizaciones a un nivel elemental. Así, la enseñanza de los prefijos debe tener en cuenta distintos componentes como el semántico, el sintáctico y el pragmático.

Palabras clave: prefijos; formación de palabras; didáctica de la lengua; enseñanza de la morfología.
Abstract: This paper examines the issues which are identified in the lists of prefixes and activities which are related to prefixes in Secondary Education books. The Spanish Language and Literature subject may provide the students with the necessary tools and knowledge which will give them the essential knowledge to manage any communicational situation. One of these knowledges are the grammar. In the following pages we discuss that the exercises of identification and labeling of prefixes and production do not improve students' ability to observe, argue, reflect, and, specifically, understand morphologic structures. Our research focuses on the use of prefixes in the Secondary Education level, throughout a variety of activities which favour the development of student's critical thinking ability to observe morphological phemomena and to create generalizations at a basic level. Thus, teaching prefixes must take into account different components like the semantic, the syntactic and the pragmatic.

Keywords: prefixes; words formation; language didactic; morphology teaching. 


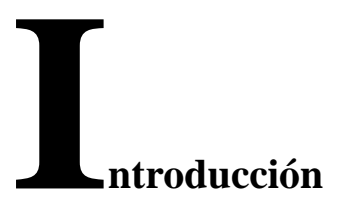

La materia de Lengua castellana y Literatura en la etapa de secundaria tiene como objetivo fundamental el desarrollo de la competencia comunicativa del alumnado en todas sus vertientes: pragmática, linguística, social y literaria (Real Decreto 1105/2014, de 26 de diciembre). Por ello, esta asignatura debe aportar las herramientas y los conocimientos necesarios para que el estudiante pueda desenvolverse en cualquier situación comunicativa. Teniendo presente este objetivo, cabe preguntarse qué papel juega la morfología en esta competencia comunicativa. Para responder esta cuestión, vamos a centrarnos en un proceso morfoléxico concreto como es la prefijación, con el fin de analizar los problemas que presentan tanto la clasificación de los prefijos como los ejercicios sobre este tema en los manuales de secundaria. La selección de editoriales empleada en este trabajo está en consonancia con el uso de los mismos en esta etapa, es decir, son manuales usados frecuentemente. El uso de varios responde a la posibilidad que brinda la comparación de varios materiales para establecer un análisis exhaustivo. A partir de los datos que se obtengan de este estudio, esbozaremos una muestra de actividades que favorezcan el desarrollo de la capacidad crítica del alumno para observar fenómenos morfológicos y formular generalizaciones a un nivel 
elemental. Son estos dos puntos los que vertebran el estudio que presentamos en este trabajo.

El tema que presentamos resulta de interés si se considera que la enseñanza de la morfología no tiene un papel importante en el aula de secundaria a pesar de los beneficios que el conocimiento morfológico proporciona en el desarrollo de la competencia comunicativa (Martín Vegas, 2018; Gil, 2020). Dentro de la enseñanza de la morfología, la prefijación es uno de los temas menos tratados, si cabe, y la información que se aporta es muy limitada y, en algunos casos, deficitaria. Por ejemplo, los ejercicios sobre prefijos se muestran como un sistema automático que consiste, principalmente, en actividades de respuesta rápida como rellenar espacios en blanco o asignar etiquetas a las palabras de un texto. Son, por ello, tareas mecánicas que no contribuyen a mejorar el conocimiento de la lengua ni a desarrollar la competencia comunicativa, en la medida en que los estudiantes no consiguen interiorizar estos conocimientos.

En los últimos años, han sido varios los trabajos que se han escrito sobre la didáctica de la gramática, en general, y sobre la didáctica de la morfología, en particular, abarcando todos los niveles de enseñanza, desde la educación primaria hasta la enseñanza no obligatoria (por ejemplo, Bosque, 2015; Martín Vegas, 2015; 2018; Bosque y Gallego, 2020; Buenafuentes, 2020; Gil, 2020). Todos ellos apuestan por la enseñanza de una gramática basada en la reflexión, metodología en la que el estudiante es el protagonista de su aprendizaje. Esta preocupación por la enseñanza de la morfología desde una perspectiva más reflexiva también se observa en el ámbito de la enseñanza de español como lengua extranjera (ELE), en trabajos como Martín García y Varela (2009), Serrano Dolader (2018), Martín García (2020), entre otros.

En este trabajo, partiremos de la hipótesis de que la enseñanza de la morfología puede desarrollarse de una forma reflexiva en el aula, aprovechando, por un lado, el conocimiento interno que posee el estudiante sobre la lengua y, por otro, la curiosidad como un factor de motivación. De este modo, se pretende que el estudiante consiga 
desarrollar estrategias descriptivas y analíticas para resolver los problemas que se le proponen.

Teniendo en cuenta este planteamiento, hemos dividido el artículo en tres apartados. En el primero, describimos las características básicas de los prefijos que, desde nuestro punto de vista, deberían considerarse en la enseñanza secundaria y que recogeremos en nuestra propuesta. En el segundo, examinamos, por un lado, la información sobre los prefijos que aportan los libros de texto de secundaria $\mathrm{y}$, por otro, los ejercicios que se proponen. Por último, presentamos una propuesta de actividades sobre la enseñanza de los prefijos en secundaria desde la reflexión. Cerramos el artículo con unas conclusiones generales.

\section{1.- Características de los prefijos}

Desde los estudios más tradicionales, no ha existido unanimidad a la hora de considerar la prefijación como un proceso de composición o de derivación. Salvo la consideración de los prefijos como morfemas adjuntados al inicio de una palabra, han sido muchas las discrepancias a la hora de fijar las características de estas unidades morfológicas. Esto ha tenido al menos dos consecuencias en la enseñanza de la morfología: por un lado, se ha prestado muy poca atención a la prefijación y, por otro, existen muchas diferencias en la información que se ofrece en los manuales. En este apartado, presentaremos las características más básicas que se asumen de forma general en los estudios de morfología y en las gramáticas del español.

La primera cuestión sobre la prefijación está relacionada con el lugar que ocupa este proceso dentro de la morfología. La gramática tradicional incluía la prefijación dentro de la composición por la semejanza entre algunos prefijos con las preposiciones (antecámara, convivir, entremezclar, sobrecubierta) (Alemany Bolufer, 1920). Dentro de la morfología léxica, se ha considerado también que la prefijación es un mecanismo derivativo, en cuanto que muchos prefijos no tienen relación con una preposición existente en la lengua 
(inmaduro, rehacer) y los prefijos con valor preposicional pueden presentar también significados no representados en la preposición (Varela y Martín García, 1999; RAE y ASALE, 2009). Así, el prefijo entre- en entremezclar tienen un valor preposicional 'mezclar entre varias cosas', pero este mismo prefijo en entreabrir exhibe un significado intensivo 'abrir un poco o a medias'. Considerar la prefijación como una parte de la composición o de la derivación afecta a la lista de prefijos que se presenta en las gramáticas y en los estudios. Una consecuencia de esta discrepancia es la diferencia en el número de prefijos que se incluyen en los manuales de enseñanza secundaria.

A diferencia de los sufijos que modifican la categoría y el significado de la base a la que se adjuntan (cuidar $>_{\mathrm{V}}^{>}$cuidador $_{\mathrm{N}}$ ), los prefijos no son elementos categorizadores, por lo que no cambian la categoría gramatical de la palabra a la que se añaden (grande $\mathrm{A}-$

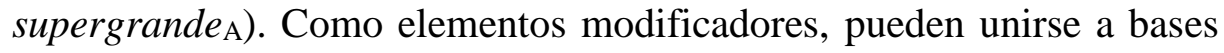
de distintas categorías: adjetivos (imborrable), nombres (exalumno), adverbios (superbién), verbos (descalificar) e, incluso, a sintagmas preposicionales (super de mal humor) y nominales (pro derechos humanos).

A partir del significado que aportan a la base, se han reconocido distintos grupos de prefijos. Por ejemplo, en la clasificación de RAE y ASALE (2009), se distinguen los siguientes grupos: espaciales (antepenúltimo, intracelular, subcontinente), temporales (anteayer, prefabricar), cuantificativos (multicolor, políglota, bianual, semicerrado), gradativos y escalares (reguapo, superordenador, hiperconocido, subdelegado, infravalorado), aspectuales (desaparecer, redecorar), negativos (imposible, desconfiar) y de orientación o disposición (antiacadémico, contraargumentación). De todos los grupos, el más productivo en el español actual es el de los prefijos gradativos, los cuales pueden aportar valores de intensificación aplicados a propiedades (archiconocido, extrafino, relimpio, superelegante) o aplicados al tamaño de una entidad (megaciudad, minitarjeta). 
Otro criterio que se ha establecido para la clasificación de los prefijos es su dependencia o independencia formal, reconociendo los prefijos inseparables de la base (es decir, de la palabra a la que se adjuntan) (preventa) y los prefijos separables (ex presidente, pro aborto, anti-OTAN) (RAE y ASALE, 2009). En el caso de estos últimos prefijos, la separabilidad conlleva cierta independencia con respecto a las reglas ortográficas.

Se ha señalado en los distintos trabajos que la polisemia es una característica de los prefijos. Por ejemplo, el prefijo re-significa 'volver a' en releer o puede aportar un matiz intensificador en relimpio ('muy limpio'). Este hecho tiene cierta importancia en el aprendizaje de estos procesos morfológicos, por cuanto que los estudiantes deben advertir estas diferencias para interpretar tanto las palabras prefijadas existentes como los neologismos.

Si nos atenemos a la forma, cabe señalar que varios prefijos presentan alomorfos (esto es, variantes formales de un mismo morfema). Por ejemplo, el prefijo in- presenta los alomorfos im- cuando se adjunta a una palabra que comienza con una consonante oclusiva bilabial (imborrable) o $i$ - delante de palabras con una consonante líquida inicial (irreal, ilegal). Como veremos en el siguiente apartado, la alomorfia no se contempla en ningún manual, lo que supone considerar las variantes alomórficas como prefijos distintos.

Relacionado también con la forma, conviene señalar que algunos prefijos forman parte de palabras heredadas del latín y no existen como morfemas en español desde una perspectiva sincrónica, como en obligar o admirar. Estos prefijos no son identificables por los hablantes de español y no dan lugar a formaciones nuevas. Asimismo, estas palabras no tienen una estructura morfológica transparente en la que sea posible identificar sus constituyentes y componer el significado. Por ejemplo, en la palabra inhumar no tenemos en español el elemento base *humar, por lo cual esta palabra presenta opacidad formal. Véase que en el caso de estos términos solo acudiendo al latín es posible dar con su significado. En el caso de inhumar, el prefijo in- con el significado locativo 'dentro de' se une al tema *humar, procedente de 
humus/-i 'tierra' y la vocal temática de la primera conjugación. Ni la base humus ni el tema humar se han materializado como una palabra en español. Unidades léxicas de este tipo son frecuentes en los libros de texto de secundaria para ejemplificar los procesos morfológicos. Sin embargo, como hemos dicho, este prefijo solo existe en palabras heredadas del latín, por lo cual su uso en la enseñanza de la morfología desde una perspectiva sincrónica es bastante discutible.

La última característica que comentaremos tiene que ver con la combinación de los prefijos y su recursividad. Los prefijos pueden seleccionar palabras previamente prefijadas (des-com-poner). Otra posibilidad es que los prefijos se coordinen (pre y poselectoral, bi $y$ tricolor). A diferencia de los sufijos, un mismo prefijo puede repetirse sin que se produzca un cambio en el significado denotativo ya que, con la reduplicación, se consigue una mayor intensidad (super-super-grande $>$ 'muy muy grande'). En otros casos, la reduplicación del prefijo supone un cambio de significado (anti-anti-taurino 'en contra de los antitaurinos').

A partir de las características señaladas, vamos a analizar en el apartado siguiente el tratamiento que reciben los prefijos en los manuales de lengua española de secundaria.

\section{2. - Los prefijos en los manuales de secundaria 2.1.- Caracterización de los prefijos}

Si se mira con detenimiento cualquier libro de texto de lengua española de secundaria, es fácil advertir que la prefijación no ocupa un lugar destacado, sino que suele aparecer incluida junto a la sufijación dentro del apartado de palabras derivadas. Así ocurre, por ejemplo, en los manuales de Oxford $1^{\circ}$ de ESO, de Anaya $2^{\circ}$ de ESO, de SM $1^{\circ}, 2^{\circ}$ y $3^{\circ}$ de ESO. Son pocos los libros de texto que separan la sufijación de la prefijación, como el manual de $\mathrm{SM} 4^{\circ}$ de ESO. A continuación, vamos a enumerar algunas inconsistencias que hemos apreciado en nuestro estudio. 
Uno de los problemas recurrentes que encontramos en los distintos manuales consultados de Lengua Castellana y Literatura es la confusión terminológica en la denominación de las unidades, lo que afecta a la hora de definir y clasificar los prefijos. En la definición de prefijo, se destaca el lugar que ocupa esta unidad morfológica en la estructura de la palabra (1a, b, c); en el caso de la prefijación, simplemente se describe el proceso $(1 \mathrm{~d}, \mathrm{e})$ :

(1) a. "Prefijos: se colocan delante del lexema: in-feliz, des-amparo" (Oxford, $1^{\circ}$ ESO)

b. "Los prefijos son morfemas derivativos que preceden al lexema: des-cansar, in-cauto, pre-decir, ante-poner". (Anaya, $\left.2^{\circ} \mathrm{ESO}\right)$

c. "Los prefijos son morfemas afijos que preceden a un morfema léxico o raíz modificando su significado y creando nuevas palabras" (SM, $4^{\circ}$ ESO)

d. "Prefijación: se añade un morfema prefijo a la raíz: des-orden, in-coherente." (SM, $\left.2^{\circ} \mathrm{ESO}\right)$

e. "La prefijación: se añade un prefijo a la base léxica." (SM, $\left.3^{\circ} \mathrm{ESO}\right)$

En las definiciones anteriores, se considera el prefijo como un morfema derivativo o como un morfema afijo, según se tenga en cuenta el proceso morfológico o el tipo de morfema. En esta diferencia está presente la distinta concepción de la morfología que asumen los autores de los libros de secundaria. Por un lado, la definición de prefijo como un morfema afijo se hace desde una perspectiva en la que existen tres tipos de morfemas: raíces (o lexemas), afijos y temas grecolatinos. Dentro de los afijos, se pueden diferenciar los prefijos, sufijos, interfijos e infijos. Por otro lado, la definición de prefijo como morfema derivativo parte del proceso en el que están implicados los morfemas: morfemas derivativos y morfemas flexivos. Las definiciones así planteadas son excluyentes, pero las dos características que se mencionan definen el prefijo, de modo que un prefijo es un afijo derivativo. Otro de los problemas terminológicos que presentan las definiciones anteriores tiene que ver con la denominación de la unidad a la que el prefijo se adjunta. Como vemos, se habla de lexema, morfema léxico, raíz y base léxica. La raíz es un morfema léxico, como lo son los afijos derivativos, que se define como la primera unidad básica que puede experimentar un proceso morfológico y que es común a una familia léxica. Con este sentido, el prefijo se une a una raíz en formaciones como superfácil, pero la definición no es válida cuando el 
constituyente al que se adjunta el prefijo es una palabra derivada (borra $(r)>$ borrable > imborrable). El mismo problema plantea el término lexema, al identificarse con raíz. La base, por su parte, alude al constituyente sobre el que opera un proceso morfológico y puede ser una raíz o una palabra derivada. Por ello, en el tipo de definición que aparece en (1), lo más conveniente es hablar de base o de base léxica.

En cuanto a la clasificación de los prefijos, la mayoría de los manuales de secundaria presentan una pequeña lista de prefijos, sin consignar los criterios seguidos en la selección. En estas listas, se observan discrepancias en el número de prefijos incluidos, como ocurre también en los trabajos descriptivos sobre prefijación. Estas diferencias se producen según se adopte una perspectiva sincrónica o diacrónica, o según se adopte un criterio selectivo o acumulativo ${ }^{1}$. La diferencia en el número de prefijos tratados también puede darse en los distintos cursos de los manuales de una misma editorial, como ocurre en los libros de texto de la editorial SM. En la tabla siguiente, se incluye el número y los prefijos considerados en cada curso:

\section{Tabla 1}

Prefijos incluidos en los libros de texto de la editorial SM

\begin{tabular}{|c|c|c|}
\hline Cursos & $\begin{array}{l}\mathrm{N} .^{\circ} \text { de } \\
\text { prefijos }\end{array}$ & Prefijos \\
\hline $1^{\circ} \mathrm{ESO} \mathrm{SM}$ & 7 & $\begin{array}{l}\text { a-, des-, extra-, i-, im-, in-, pre-, re-, } \\
\text { sub- }\end{array}$ \\
\hline $2^{\circ} \mathrm{ESO} \mathrm{SM}$ & 12 & $\begin{array}{l}\text { a-, an-, anti-, co-, con-, contra-, des-, } \\
\text { ex-, extra-, i-, in-, inter-, pre-, re-, sub- }\end{array}$ \\
\hline $3^{\circ} \mathrm{ESO} \mathrm{SM}$ & - & - \\
\hline $4^{\circ} \mathrm{ESO} \mathrm{SM}$ & 23 & $\begin{array}{l}\text { a-, an-, anti-, contra-, des-, dis-, en-, } \\
\text { entre-, ex-, extra-, i-, in-, inter-, intra-, } \\
\text { pos(t)-, pre-, pro-, re-, requete-, sub-, } \\
\text { tras- }\end{array}$ \\
\hline
\end{tabular}

Fuente: elaboración propia

\footnotetext{
${ }^{1}$ En los distintos trabajos dedicados a la prefijación, las diferencias en el número de prefijos considerados pueden ser llamativas. De hecho, el número de prefijos puede oscilar entre los veintiocho prefijos que reconoce Lang (1992) y los doscientos dos que incluye el trabajo de Rainer (1993).
} 
En una primera aproximación, podría pensarse que, en esta tabla, se sigue un método acumulativo, según el cual se incluyen nuevos prefijos en cada curso. De este modo, el curso superior tiene más prefijos que los cursos inferiores. Sin embargo, es fácil apreciar que algunos prefijos se repiten y otros se añaden por primera vez, sin que aparentemente haya un criterio claro que justifique esta selección. Por ejemplo, en el curso de $2^{\circ}$ de ESO se incluyen como prefijos co-/con- y pre-, pero estos mismos prefijos se excluyen en el curso de $4^{\circ} \mathrm{ESO}$. Si se sigue un método acumulativo, los prefijos del curso inmediatamente anterior deberían incluirse en el siguiente, junto con los nuevos prefijos. Además, como podemos observar en la tabla, el manual de $3^{\circ}$ de ESO no ofrece ningún inventario sobre prefijos, simplemente aporta dos ejemplos en la explicación del proceso de prefijación (impuro y subrayar).

La tabla anterior plantea más problemas que traslucen ciertos errores en la concepción de la morfología. El primero tiene que ver con los alomorfos, interpretados como prefijos distintos. Así, en el libro de $4^{\circ}$ de ESO, requete- (requetealto) es considerado como un prefijo distinto de re-(reguapo), cuando los dos son alomorfos de un mismo prefijo intensivo. Lo mismo ocurre con el alomorfo $i$ - (irreal), tratado como un prefijo distinto de in- (inmaduro). En este caso, curiosamente, no se ha incluido el alomorfo im- (impuro), que es más productivo que el alomorfo $i$-. Tampoco parece que haya una explicación clara que justifique incluir inter-, pero no entre-, cuando son dos alomorfos del mismo prefijo: inter- es la variante culta y entre-, la variante patrimonial (interdisciplinarlentrecomillar) (RAE y ASALE, 2009, p. $688)$.

Otro problema que se observa es la inclusión de alomorfos del latín que ya no son productivos en el español actual, como el prefijo dis- (discordia), alomorfo no productivo del prefijo des- (deshonra). En este caso, además de considerar esta forma como un prefijo, es una variante que solo existe en las palabras heredadas en latín porque no ha generado ninguna formación en español. Si la enseñanza de la 
morfología en secundaria debe adoptar una perspectiva sincrónica, estas unidades no pueden estar listadas junto con prefijos productivos.

Además de las listas de prefijos, los manuales facilitan ejemplos de palabras prefijadas. Tampoco esta práctica está libre de problemas. Como puede observarse en los manuales, junto con formaciones que presentan una transparencia formal, en el sentido de que pueden reconocerse los constituyentes, aparecen otras palabras opacas formalmente. Por ejemplo, en una palabra como reducir se reconoce el prefijo re- por recurrencia con otras palabras (rehacer, reconstruir, reaparecer), pero también un segundo constituyente que se repite en otras palabras (producir, introducir...). El problema reside en el hecho de que el constituyente -ducir no es una palabra en español, lo que dificulta la construcción del significado. En este caso, la falta de transparencia formal va asociada a una opacidad semántica. El verbo reducir no tiene un significado composicional y no admite la paráfrasis iterativa propia del prefijo $r e$ - presente en verbos como rehacer ('volver a hacer') o reconstruir ('volver a construir'). Como hemos mencionado, en la enseñanza de la morfología debe adoptarse una perspectiva sincrónica, que permita identificar la estructura de las palabras complejas, a partir de la cual sea posible componer el significado de las palabras. Por tanto, palabras como reducir deben estar excluidas como ejemplos de palabras derivadas. Asimismo, puede darse opacidad semántica en palabras en las que es posible reconocer sus constituyentes. Por ejemplo, el verbo repartir está formado con el prefijo re- y el verbo partir, pero su significado no es 'volver a partir' (como en rehacer) sino 'distribuir en varias partes', es decir, el significado no es predecible a partir de los constituyentes que integran la palabra.

Como hemos dicho, en los manuales de secundaria solo se ofrece información sobre el significado de los prefijos, pero no se contempla la polisemia. Por ejemplo, el prefijo re- está clasificado en el grupo de los prefijos gradativos en el manual de $4^{\circ}$ ESO (reguapo, remirar), pero este mismo prefijo es más productivo en español como prefijo iterativo (reordenar, reescribir), significado que está excluido de los manuales. 
También sorprende comprobar que, siendo el grupo de los prefijos gradativos el más productivo en español y el que más prefijos incluye, solo se mencione el prefijo re- y su alomorfo requete-. Se excluyen otros muchos prefijos intensivos, algunos más productivos que re-: archi-, hiper, infra-, macro-, mega-, micro-, re-, semi-, sobre-, sub-, super-, supra- o ultra-. Además, la definición que se ofrece sobre estos prefijos no se ajusta a los prefijos que se dan como modelo. Por ejemplo, según el manual de $4^{\circ}$ de ESO de SM "los prefijos gradativos miden el grado de una propiedad o expresan la identidad de una acción" (Blecua, 2016, p. 160). Dependiendo de si la base es un sustantivo o un adjetivo, algunos prefijos bien pueden medir el grado de una propiedad en un adjetivo, o bien pueden intensificar las cualidades que representa la base nominal: por ejemplo, un supermóvil denota un móvil que posee unas características o cualidades de grado superior, tales como la capacidad, la potencia, la rapidez, entre otras. Además, estos prefijos también pueden aludir al tamaño, a la cantidad o a la cualidad de la palabra intensificada y dan lugar a formaciones con cierta ambigüedad: superpiso puede hacer referencia al tamaño (piso de grandes dimensiones) o a las propiedades intensificadas (piso extraordinario).

Otro de los problemas que observamos en los manuales de secundaria es la confusión entre prefijo, elemento compositivo y acortamiento. Por ejemplo, en algunos manuales, unidades como macro- o micro- (microorganismo en el manual de $4^{\circ}$ de ESO de SM) o seudo- son considerados como elementos compositivos y, por tanto, están tratados dentro de la composición. En las gramáticas de español y en los trabajos dedicados a la prefijación, estos elementos son analizados como prefijos. Por otro lado, algunos prefijos coinciden formalmente con un acortamiento, como ocurre con auto-, que puede ser un prefijo (autovacuna) o el acortamiento de automóvil (autoescuela). Lo mismo ocurre con el prefijo tele- (teletrabajo, teleclase, teledirigir) y el acortamiento de televisión, presente en formaciones como telebasura, telemaratón. Estas distinciones no se establecen claramente porque, como ocurre con los acortamientos, se alude rara vez a este tipo de nociones. 
Por último, resulta llamativo que se hable de prefijos aspectuales en el manual de SM de $4^{\circ}$ de ESO cuando en ninguna parte del libro de texto se ha hablado del aspecto léxico de los verbos. Según Varela y Martín García (1999: 5028), los prefijos aspectuales afectan a la acción verbal modificando el aspecto del verbo (des-, re-, sobre-, sub-). No obstante, los prefijos aspectuales que se ofrecen en el manual de secundaria de SM son solamente ex- y re-, sin ofrecer ningún ejemplo, por lo que el prefijo re-, que está clasificado en el grupo de los prefijos aspectuales, puede confundirse con el prefijo gradativo re-.

En resumen, no parece que sea viable hacer una clasificación tan reducida y escasa a partir del significado que aportan los prefijos². Además, como hemos comentado, en los libros de texto de secundaria se incluye información no relevante como los alomorfos no productivos en el español actual (dis-) y, al mismo tiempo, se excluye información importante como los alomorfos de los prefijos (in-, im-). Así pues, una vez analizados los manuales, podemos llegar a la conclusión de que la selección de prefijos no sigue unos criterios claros ni lógicos, como serían la productividad y la frecuencia de los prefijos, por ejemplo.

\section{2.- El tratamiento de los prefijos en los ejercicios de los manuales de secundaria}

Los ejercicios de prefijación que aparecen en los manuales de secundaria suelen ser en su mayoría de respuesta corta, dejando de lado la argumentación o la comprensión de los fenómenos gramaticales que se tratan. Gallego (2016, p. 146) señala que los ejercicios de gramática en los libros de texto de secundaria son ineficaces y, además, prescinden de la utilidad y de la comunicación oral habitual. En este sentido, la tipología de ejercicios que se sigue en estos manuales resulta inadecuada si el objetivo que se pretende perseguir es la reflexión lingüística de los discentes (Buenafuentes, 2020). Los ejercicios que comentamos en este apartado tienen que ver con el reconocimiento de las formas, sin incluir la reflexión. En los distintos manuales de

\footnotetext{
2 Podemos concluir que la selección de prefijos en los libros de texto es inadecuada. No obstante, para estudiar una clasificación más adecuada sobre los prefijos, podemos recurrir a trabajos como RAE y ASALE (2009) y Varela y Martín García (1999).
} 
secundaria, podemos diferenciar al menos dos tipos de ejercicios relacionados con la morfología y, más concretamente, con la prefijación: por un lado, los ejercicios de reconocimiento, que a su vez se subdividen en ejercicios de identificación de constituyentes y en ejercicios de etiquetado, y, por otro, los ejercicios de producción.

En los ejercicios de reconocimiento, encontramos actividades de identificación de constituyentes, donde el estudiante debe simplemente localizar los prefijos o las palabras prefijadas, como aparece en los ejemplos de (2):

(2) Ejercicios de reconocimiento e identificación de constituyentes:

(a). ¿Alguna palabra contiene un prefijo? [p. 19] (SM, Lengua Castellana, $\left.3^{\circ} \mathrm{ESO}\right)$.

(b). Identifica en el texto una palabra derivada mediante un prefijo [p. 18] (Anaya, Lengua Castellana, $2^{\circ} \mathrm{ESO}$ ).

(c). Localiza los prefijos y sufijos de este cartel [p. 161] (SM, Lengua Castellana, $4^{\circ}$ ESO).

Como refleja la instrucción de estos ejercicios, este tipo de tarea no favorece la reflexión por parte del estudiante. Son actividades mecánicas en las que el estudiante no analiza las formas ni detecta el valor semántico que aporta un prefijo a la base a la que se añade. Tampoco se plantea ningún problema o reto que el estudiante deba superar. Además, las palabras que se incluyen para ser identificadas presentan cierta opacidad formal y semántica, lo que impide que el estudiante pueda construir hipótesis que le permitan entender los procesos morfológicos. En primer lugar, algunas palabras carecen de estructura morfológica, ya que no es posible reconocer los constituyentes que las componen. Por ejemplo, en la actividad (2a) se muestra un cartel universitario en el que el estudiante tiene que buscar las palabras prefijadas ecológica, desplazarte y sostenible ${ }^{3}$. No obstante,

${ }^{3}$ El significado de desplazar no significa 'no plazar' ni sostenible significa 'por debajo de lo tenible'. En este último caso, el significado locativo de la forma $s o(s)$-, alomorfo del prefijo $s u b$-, solo aparece en palabras heredadas del latín en las que el significado 
ninguna de estas palabras ha sido formada mediante el proceso derivativo de prefijación en el español actual. Por ejemplo, la palabra ecológica se genera desde el tema grecolatino eco-. En los otros casos, para analizar las palabras desplazarte o sostenible el estudiante necesita adoptar una perspectiva diacrónica y aplicar unos conocimientos históricos de la lengua que no tiene. Ocurre lo mismo en la actividad (2b), donde el estudiante debe identificar una palabra derivada por un prefijo en un texto, las palabras prefijadas son: invisibles, descubierto e influyera. Estas dos últimas presentan el mismo problema que el ejercicio anterior, ya que tanto descubrir como influir no son transparentes ni en la forma ni en el significado. Por último, en la actividad (2c) la única palabra prefijada que aparece es intolerancia, por lo que el estudio de los prefijos en este ejercicio es insuficiente si el estudiante debe localizar, simplemente, un solo caso.

Otro tipo de actividades son los ejercicios de etiquetado. En (3) se muestra la instrucción de analizar, ${ }^{4}$, pero no se especifica el tipo de análisis, por lo que simplemente con el enunciado no es posible saber si se refiere a un análisis morfológico, sintáctico o semántico.

(3) Analiza los prefijos y sufijos de las palabras destacadas. [p. 160] (SM, Lengua Castellana, $4^{\circ}$ ESO).

Las palabras destacadas que se deben analizar en (3) son: postelectoral, destapar y envasada. El objetivo de este ejercicio es que los alumnos segmenten los prefijos y los sufijos e indiquen el significado de cada uno. Si comprobamos la solución de los ejercicios en el libro del profesor, advertimos que los análisis que se proponen son imprecisos. Por ejemplo, en el solucionario se especifica que el prefijo en- indica movimiento. Sin embargo, en el ejemplo envasada el prefijo expresa la locación (literalmente, envasar es 'meter algo en un vaso'). El prefijo des- en destapar no indica lo contrario, tal y como se refleja

no es composicional. Además, sos- no es un alomorfo recurrente ni productivo en el español actual.

${ }^{4}$ El objetivo de analizar es entender y explicar el significado de las palabras. Sin embargo, en los ejercicios de gramática, analizar consiste simplemente en etiquetar, por lo que los ejercicios de etiquetado se convierten en el principio y en el final del análisis (Bosque y Gallego, 2020). 
en el manual del profesor, puesto que destapar no significa 'no tapar', sino la reversión de un estado ('hacer que algo que estaba tapado deje de estarlo'). Por último, para detectar el valor de posterioridad del prefijo post- en postelectoral ('que va después de las elecciones'), es mejor recurrir a un sustantivo como posromanticismo ('época posterior al Romanticismo'). Por tanto, estas soluciones imprecisas que aparecen en el solucionario pueden confundir a los estudiantes y al profesor.

Por otro lado, encontramos en los manuales ejercicios de producción, en los que el estudiante debe crear palabras mediante la unión de prefijos y sufijos, como vemos en (4):

(4) Relaciona las palabras de la izquierda con los prefijos y sufijos, creando el mayor número de palabras posibles. (SM, Lengua Castellana, $4^{\circ}$ ESO).

Las palabras que ofrece el ejercicio (4) son deporte, cultura, sensible, cumplir y moderno, y los prefijos que presenta son post-, in- y anti-. Con este número de palabras y el número de prefijos, el estudiante podrá formar tan solo seis palabras: antideporte, incultura, insensible, incumplir, postmoderno, antimoderno. Este tipo de producción es muy limitada y no tiene un objetivo más allá de la formación de la palabra. Además, si el estudiante no conoce la palabra en cuestión, no podrá crearla porque no se aporta ningún mecanismo que facilite este proceso. Por otro lado, tampoco hay una relación entre las palabras que el estudiante debe crear (por ejemplo, palabras con el mismo prefijo o con prefijos que pertenezcan al mismo tipo). Además, los procesos productivos aparecen mezclados con los no productivos: así, el prefijo in- unido a nombres es muy productivo (incultura), frente al prefijo in- unido a verbos (incumplir). Por tanto, con este tipo de actividades, el estudiante no aprende nada nuevo y resulta imposible que pueda generar hipótesis y llegar a conclusiones. Para que la producción sea realmente una actividad de aprendizaje, debe estar basada en procesos concretos, de modo que a partir de las palabras creadas el estudiante pueda reflexionar sobre el patrón de formación. 
Otra posibilidad en las actividades que se proponen es la combinación de ejercicios de reconocimiento y de análisis, como vemos en (5):

(5) Localiza dos palabras que tengan un prefijo y analiza su estructura [p. 161] (SM, Lengua Castellana, $4^{\circ}$ ESO).

En el ejercicio (5), se presenta un texto en el que el estudiante debe identificar los prefijos para analizar la estructura de la palabra. Los ejemplos que se ofrecen son requeteguapa y requetepequeñuela, con el alomorfo requete-, poco productivo en español. Para intensificar este tipo de adjetivos, disponemos de otros prefijos gradativos mucho más productivos, como hiper-, super-o mega-. De este modo, una actividad así planteada no ofrece ningún tipo de profundización, porque no se pide al estudiante ninguna justificación de su análisis: ¿por qué se utiliza determinado prefijo?, ¿qué significado aporta a la formación? o ¿qué diferencia hay con otros prefijos, por ejemplo, entre requeteguapa y superguapa?

Como hemos comentado, los ejercicios no están orientados a la descodificación de los morfemas con el fin de que los estudiantes logren una comprensión lingüística ni a la codificación del significado mediante los morfemas para crear nuevas palabras, objetivos que se ignoran frecuentemente en la didáctica de la morfología, según señala Buenafuentes (2020).

En suma, tanto los ejercicios de identificación de constituyentes como los ejercicios de etiquetado no son favorables para el desarrollo de la competencia lingüística del estudiante, porque no facilitan una explicación o una argumentación. Como hemos visto, la mayoría de estos ejercicios de prefijación en secundaria consisten en recordar el material aprendido con anterioridad desde primaria, como el significado de los prefijos que ya conocen como hablantes de la lengua. Estos ejercicios son mecánicos y no aportan conocimientos nuevos. En este sentido, puede afirmarse que no existe una progresión del conocimiento. 


\section{3.- Muestra de actividades}

En este apartado se presenta un modelo de actividades que pueden realizarse en las sesiones de morfología de secundaria, con el objetivo de que el alumno tenga un papel activo en su aprendizaje. Para ello, las actividades que se planteen deben ser variadas y motivadoras, de modo que el alumno sienta cierto interés y curiosidad por los hechos lingüísticos que se tratan. Además, se pretende que el estudiante sea capaz de entender y explicar los procesos morfológicos a partir de los datos que recibe, es decir, se trata de que el estudiante desarrolle su capacidad crítica, no solo acumulativa.

La enseñanza de la morfología debe basarse en el reconocimiento de las formas, en la construcción del significado a partir de los constituyentes que integran una palabra, en la producción y uso de las formaciones complejas, así como en la distinción entre pares semejantes (Buenafuentes, 2020 y Gil, 2020). Las actividades de reflexión en la enseñanza de la gramática aportan al estudiante la capacidad de examinar las relaciones que existen entre la forma y el sentido (Bosque, 2018), bien desde un planteamiento inductivo o deductivo. Este tipo de actividades donde se combina el análisis morfológico con el semántico y el sintáctico permiten al estudiante explicar los procesos morfológicos a partir de los datos que recibe, al mismo tiempo que pone en relación competencias de distintos ámbitos lingüísticos.

La muestra que presentamos se articula en tres ejes: el reconocimiento de los elementos derivados de una palabra, la composición del significado a partir de los constituyentes y el uso de la formación compleja en contextos comunicativos adecuados.

Para las actividades de reflexión se han propuesto varios tipos de tareas como pares mínimos, análisis inverso o análisis con secuencias agramaticales (Bosque y Gallego, 2016). Este tipo de actividades son válidas para la enseñanza de la morfología, como se ha puesto de relieve en trabajos dedicados a este tema (por ejemplo, Buenafuentes, 2020 y Gil, 2020). A partir de estos tipos de ejercicios, es posible 
diseñar actividades destinadas a la enseñanza de los prefijos. El primer tipo que comentaremos es el análisis directo a través de preguntas encadenadas que guían al estudiante en la construcción del conocimiento. El segundo tipo de actividades está basado en la composición de significado de la palabra a través de los constituyentes. El tercer tipo que proponemos para trabajar los prefijos son ejercicios basados en el análisis inverso, esto es, el estudiante recibe dos o tres propiedades que debe formalizar en un ejemplo. Por último, mostramos una secuencia de ejercicios de pares mínimos donde el alumno debe explicar propiedades gramaticales concretas.

En primer lugar, la actividad que proponemos está basada en analizar secuencias breves cuyo objetivo es distinguir aspectos del análisis que no son evidentes a priori. En (6) se presentan dos palabras prefijadas con $r e$ - en un contexto dado y, a continuación, se ofrece una secuencia de preguntas.

(6)

El prefijo re-puede aportar distintos significados a la palabra a la que se adjunta. Observa las palabras en cursiva de las siguientes oraciones:

(1) Puedes redecorar la habitación.

(2) Puedes rebuscar en la habitación.

a) ¿Qué diferencia hay entre (1) y (2)?

b) ¿Cuál es el significado que aporta $r e$ - en cada caso?

c) Encuentra al menos tres palabras prefijadas con re- en las que dicho prefijo tenga el mismo significado que en (1).

En segundo lugar, los ejercicios basados en la composición del significado tienen como objetivo que el estudiante sea capaz de deducir el significado a partir de los constituyentes de una palabra prefijada. Un buen recurso para conseguir este fin en morfología es acercar a los estudiantes a un léxico más habitual como son los neologismos. 
(7)

El prefijo super- puede unirse a muchas palabras como superdelgado, superfeo, supercoche, supermóvil, superquerer, superpintar y muchas más que habrás oído. Fíjate en estas palabras y explica qué significado tiene el prefijo super-.

Ahora agrupa las palabras anteriores según su categoría gramatical. ¿Cambia el significado del prefijo dependiendo de la categoría gramatical a la que se adjunta?

En tercer lugar, la actividad que se presenta en (8) se basa en el análisis inverso, cuyo fin es el desarrollo de la capacidad de abstracción mediante la creación de ejemplos. El estudiante debe formar ejemplos según las características especificadas. Después, debe ser capaz de reflexionar sobre los ejemplos que ha creado.

(8)

Forma palabras que cumplan las siguientes condiciones.

a. re $+\mathrm{V}$, con un significado de repetición de la acción.

b. super $+\mathrm{V}$, con un significado de intensidad.

Ahora busca contextos en los que puedan usarse los verbos que has creado. ¿Pueden usarse el prefijo re- y el prefijo super- en los mismos registros?

Por último, presentamos tres actividades con pares mínimos, en los que aparece la misma base, pero con distinto prefijo. El objetivo es que el estudiante advierta la diferencia entre palabras relacionadas y sepa distinguir el significado de prefijos aparentemente semejantes.

¿Qué diferencia hay entre los sintagmas nominales de a) y b)? Ten en cuenta la forma de las palabras y su significado.

a) El apartamento deshabitado

b) El apartamento inhabitado 
(10)

Fíjate en los siguientes ejemplos y trata de encontrar alguna diferencia en la forma y en el significado:
(1) Postmoderno
(2) Antimoderno

Intenta encontrar dos contextos comunicativos en los que se utilice cada palabra.

Observa los siguientes sintagmas nominales. ¿Tiene el mismo significado perdido? ¿Por qué?

a. Móvil perdido

b. Pueblo perdido

Si has dado con el significado adecuado, podrás explicar por qué es agramatical *móvil superperdido y no lo es pueblo superperdido.

En suma, la muestra de actividades presentada incluye ejercicios sobre la prefijación adoptando una metodología reflexiva, en la cual el estudiante puede poner en práctica lo aprendido a la vez que explica los fenómenos gramaticales. De este modo, es posible afianzar los conocimientos adquiridos. Asimismo, se exige al estudiante una actitud responsable en la construcción de su conocimiento, aprovechando la curiosidad y la motivación en la que debe basarse todo aprendizaje para que sea eficaz.

\section{Conclusiones}

En este trabajo, hemos pretendido mostrar las carencias de la enseñanza de la morfología, centrándonos en la prefijación. Para ello, hemos analizado tanto el componente teórico como los ejercicios sobre prefijación que aparecen en los libros de texto de secundaria. Hemos intentado mostrar la poca utilidad que tienen estos ejercicios en la enseñanza de la morfología, ya que las actividades que se plantean son mecánicas y no conducen al alumno a desarrollar su capacidad de análisis. 
En el último apartado del artículo, se ha incluido una muestra de actividades que favorecen el desarrollo de la capacidad crítica del estudiante a la hora de observar fenómenos morfológicos. Estos ejercicios pretenden que el estudiante aprenda a través de la reflexión, para realizar formulaciones generales a partir de los datos de los que dispone. Por último, consideramos que una enseñanza basada en la reflexión puede ayudar al estudiante a reconocer la estructura de las palabras y a componer su significado, siendo consciente de cómo funcionan los mecanismos morfológicos de su lengua.

\section{Referencias bibliográficas}

Alemany Bolufer, J. (1920). Tratado de la formación de palabras en la lengua castellana. La derivación y la composición. Estudio de los prefijos y sufijos empleados en una y otra. Madrid: Victoriano Suárez.

Bosque, I. (2015). Nuevas reflexiones sobre la enseñanza de la gramática: actitudes frente a contenidos. II Jornadas GrOC. Barcelona: UAB.

Bosque, I. (2018). Qué debemos cambiar en la enseñanza de la gramática. Revista de Gramática Orientada a las Competencias, 1(1), 11-36.

Bosque, I., y Gallego, Á. (2020). La terminología gramatical en Secundaria y Bachillerato. Ventajas e inconvenientes didácticos de algunos conceptos gramaticales antiguos y modernos. Revista Española de Lingüística, 1-29.

Blecua, J.M. (Coord.). (2015). Lengua Castellana y Literatura. $1^{\circ}$ ESO. Madrid: SM.

Blecua, J. M. (Coord.). (2016). Lengua Castellana y Literatura. $2^{\circ}$ ESO. Madrid: SM.

Blecua, J. M. (Coord.). (2016). Lengua Castellana y Literatura. $3^{\circ}$ ESO. Madrid: SM.

Blecua, J. M. (Coord.). (2016). Lengua Castellana $y$ Literatura. $4^{\circ}$ ESO. Madrid: SM. 
Buenafuentes, C. (2020). Morfología y pares mínimos: algunos ejemplos para su implantación en Secundaria. Revista de Gramática Orientada a las Competencias, III, 1-17.

Gallego, Á. (2016). Sobre los ejercicios de «reflexión gramatical»: ventajas metodológicas y aplicaciones didácticas. Revista Española de Lingüística, 46(1), 145-158. doi: https://doi.org/10.31810/RSEL.46.1

Gil Laforga, I. (2020). El análisis morfológico en el aula. Perspectiva, contenidos y actividades. Revista de Gramática Orientada a las Competencias, II(1), 43-66.

González Bernal, J. M., González Lavado, B., y Portugal Pardo, J. (2015). Lengua Castellana y Literatura $1^{\circ}$ ESO, Madrid: Oxford.

Gutiérrez, S., Luna, R., Pérez, D., y Serrano, J. (2017). Lengua y Literatura $2^{\circ}$ ESO. Madrid: Anaya.

Lang, M. F. (2009). Formación de palabras en español. Morfología derivativa productiva en el léxico moderno. Madrid: Cátedra.

Martín García, J., y Varela, S. (2009). La prefijación en E/LE: prefijos verbales. Morfología y español como lengua extranjera $(E / L E)$ (pp.67-90).

Martín García, J. (2020). La integración de contenidos morfológicos en la enseñanza de español como lengua extranjera: las nominalizaciones deverbales. Revista Internacional de Lenguas Extranjeras, 13, 1-20. doi: https://doi.org/10.17345/rile13

Martín Vegas, R.A. (2015). La didáctica de la Lengua y la Literatura Españolas en el Máster en Formación del Profesorado de Educación Secundaria. Revisión y renovación. Revista española de pedagogía, 261, 365-379. Recuperado de: https://revistadepedagogia.org/lxxiii/no-261/la-didactica-de-la-lenguay-la-literatura-espanolas-en-el-master-en-formacion-del-profesoradode-educacion-secundaria-revision-y-renovacion/101400002739/

Martín Vegas, R.A. (2018). Modelos de aprendizaje léxico basados en la morfología derivativa. Rilce: Revista de Filología Hispánica, 34.1, 262-285. doi: https://doi.org/10.15581/008.34.1.26285 
RAE y ASALE (2009). La prefijación. Nueva gramática de la lengua española. Vol. 1 Morfología. Sintaxis I (pp.663-733). Madrid: Espasa Libros.

Rainer, F. (1993). Spanische Worthildungslehre, Tubinga: Niemeyer.

Real Decreto 1105/2014, de 26 de diciembre, por el que se establece el currículo básico de la Educación Secundaria Obligatoria y del Bachillerato. Recuperado de: https://www.boe.es/boe/dias/2015/01/03/pdfs/BOE-A-2015-37.pdf

Serrano-Dolader, D. (2018). Formación de palabras y enseñanza del español LE/L2, Londres/Nueva York: Routledge.

Varela, S., y Martín García, J. (1999). La prefijación. En I. Bosque y V. Demonte (Coords.), Gramática descriptiva de la lengua española V.3 (pp.4993-5040). Madrid: Espasa Calpe, 